\title{
O uso da acupuntura antes, durante e após a gestação: Uma revisão integrativa
}

\author{
The use of acupuncture before, \\ during and after the pregnancy: \\ An integrative review
}

FisiSenectus . Unochapecó Ano 5, n. 2 - Jul/Dez. 2017 p. $13-26$

Aniele da Costa. anicosta@unochapeco.edu.br Fisioterapeuta. Especialista em Acupuntura pela Faculdade de Tecnologia Ibrate.

Sandra Silvério - Lopes. posgraduacaoacup@ibrate.edu.br Graduada em Farmácia e Bioquímica e Fisioterapia. Mestra em Tecnologia em Saúde pela Pontifícia Universidade Católica do Paraná (PUC-PR). Doutora em Ciências do Desporto pela Universidade de Trásos-Montes e Alto Douro (UTAD). Docente e Coordenadora do Curso de Pós-Graduação Lato Sensu em Acupuntura da Faculdade de Tecnologia Ibrate.

Vanessa da Silva Corralo.vcorralo@unochapeco.edu.br Farmacêutica. Mestra e Doutora em Bioquímica Tóxicológica pela Universidade Federal de Santa Maria (UFSM). Pós-Doutora também pela UFSM. Professora do Programa de Pós-Graduação Stricto Sensu em Ciências da Saúde da Universidade Comunitária da Região de Chapecó (UNOCHAPECÓ).

Clodoaldo Antônio De Sá. clodoaldo@unochapeco.edu.br

Graduado em Educação Física pela Universidade Federal de Santa Maria (UFSM). Mestre e Doutor em Ciência do Movimento Humano pela UFSM. Pós-Doutor pela Universidade de Córdoba - Espanha. Coordenador do Programa de Pós-Graduação Stricto Sensu em Ciências da Saúde da Universidade Comunitária da Região de Chapecó (UNOCHAPECÓ).

Sedinei Lopes Copatti. sedinei@unochapeco.edu.br Graduado em Educação Física licenciatura plena pela Universidade Comunitária da Região de Chapecó (Unochapecó), Especialista em Fisiologia do Exercício pela Universidade Federal do Paraná (UFPR), Mestre em Ciências da Saúde pela Universidade Comunitária da Região de Chapecó (Unochapecó).

Sabrina Lencina Bonorino.sasalencina@unochapeco.edu.br Graduada em Educação Física pela Universidade Luterana do Brasil, Mestra em Ciências da Saúde pela Universidade Comunitária da Região de Chapecó (Unochapecó).

\section{Resumo}

Introdução: Nos últimos anos, as práticas integrativas e complementares têm sido propostas como possibilidades de intervenção sobre as intercorrências associadas à gestação. Dentre as diversas técnicas, a acupuntura tem alcançado cada vez mais respaldo no ocidente. Objetivo: Analisar as produções científicas sobre o uso da acupuntura antes, durante e após a gestação, considerando os efeitos dessa intervenção. Materiais e Métodos: Revisão integrativa sobre o uso da acupuntura no período pré, durante e pósgestacional, realizada em dezembro de 2016 nas bases de dados da Biblioteca Virtual em Saúde (BVS), através do cruzamento dos descritores: "Acupuntura" or "Medicina Tradicional Chinesa" and "Gestação" or "Gravidez" or "Obstetrícia", nos idiomas português e inglês, publicados no período de 2000 a 2016. Resultados: Foram analisados 23 estudos que avaliaram o uso da acupuntura no tratamento da dor pélvica e lombar, infertilidade e fertilização, náuseas e vômito, ansiedade, depressão e insônia, além dos efeitos adversos como aborto e indução ao parto. Conclusão: A acupuntura é uma alternativa eficaz no tratamento de diversas afecções durante a gestação, porém são necessários mais estudos para comprovar seus potenciais efeitos.

\section{Palavras-chave}

Acupuntura; Gestação; Eventos adversos. 


\begin{abstract}
Introduction: In recent years, integrative and complementary practices have been proposed as possibilities for intervention on the intercurrences associated with gestation. Among the various techniques, acupuncture has been increasingly supported in the West. Objective: To analyze the scientific productions about the use of acupuncture before, during and after gestation, considering the effects of this intervention. Materials and methods: Integrative review on the use of acupuncture in the pre, during and post-gestational period, performed in December 2016 in the databases of the Virtual Health Library (VHL), by cross-referencing "Acupuncture" or "Traditional Medicine Chinese" and "Gestation" or "Pregnancy" or "Obstetrics", in Portuguese and English, published between the years 2000 and 2016. Results: Twenty-three studies evaluated the use of acupuncture in the treatment of pelvic and lumbar pain, infertility and fertilization, nausea and vomiting, anxiety, depression and insomnia, as well as adverse effects such as abortion and induction of labor. Conclusion: Acupuncture is an effective alternative in the treatment of various conditions during pregnancy, but more studies are needed to prove its potential effects.
\end{abstract}

\title{
Keywords
}

Acupuncture; Gestation; Adverse events.

\section{Introdução}

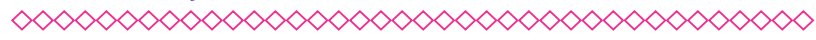

A gestação é um fenômeno fisiológico natural da mulher; entretanto, muitas vezes ocorrem dificuldades com a reprodução devido a alterações anatômicas e fisiológicas do aparelho reprodutor, acarretando infertilidade ${ }^{1}$. Já no contexto gestacional, a gravidez traz inúmeras mudanças anatômicas, posturais e fisiológicas ao organismo da mulher, as quais estão associadas a episódios de desconforto, como: dores lombares e hipogástricas, câimbras, náuseas, vômitos, refluxo gastroesofágico, constipação, síncope, tonturas, varizes, edema em membros inferiores, déficit do retorno venoso, polaciúria por redução da capacidade vesical, dentre outros ${ }^{2}$. De maneira geral, no período de gestação, as abordagens terapêuticas convencionais são escassas devido à possibilidade de complicações em relação ao feto ${ }^{3}$.

Nesse cenário, as práticas integrativas e complementares (PICs) têm sido propostas como possibilidades de intervenção sobre as intercorrências associadas à gestação, evidenciando o uso da acupuntura, que tem alcançado cada vez mais respaldo dentre as diversas técnicas da Medicina Tradicional Chinesa (MTC).

Os preceitos da MTC esclarecem que o útero é o mais importante dos seis órgãos yang extraordinários, e tem a função de regular a menstruação, a concepção, abrigar e nutrir o feto durante a gravidez. E, para isso, precisa da nutrição da essência dos rins (jing) e do sangue (Xue) ${ }^{4}$. De forma que, uma gestação somente ocorre dentro da normalidade quando os canais energéticos conseguem nutrir com abundância a essência do rim $(R)^{(4)}$.

Os sinais e sintomas referentes a uma afecção na MTC têm significados diferentes em relação à medicina ocidental, partindo do seguinte princípio: "uma doença, muitos padrões; um padrão, muitas doenças". Dessa forma, na avaliação tradicional chinesa, sintomas como vômito e náusea no início da gravidez, presença de dor, depressão pós-parto e infertilidade, advém de alterações que desestabilizam a energia vital circundante nos meridianos e órgãos, acarretando padrões energéticos que geram doença ${ }^{5}$.

A acupuntura é um antigo método chinês que se baseia na estimulação de acupontos com agulhas filiformes a fim de restaurar ou manter a energia circundante nos meridianos. As técnicas de inserção de agulhas promovem a mobilização, a circulação e o fortalecimento das energias, bem como a expulsão de deficiências ou energias perversas que acometem o indivíduo, estas que, por sua vez, são condicionantes do processo de adoecimento ${ }^{3}$.

Por outro lado, a utilização das PICS como a acupuntura, traz uma série de questionamentos, tanto em relação aos seus reais benefícios quanto 
em relação aos possíveis efeitos adversos. Com base nesses pressupostos, objetivou-se analisar as produções científicas sobre o uso da acupuntura antes, durante e após a gestação, considerando os efeitos dessa intervenção.

\section{Materiais e métodos}

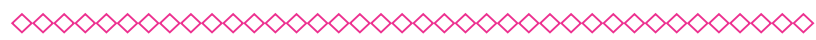

0 presente estudo utiliza como método a revisão integrativa da literatura, cujo principal foco foi proporcionar uma síntese do conhecimento em relação à utilização da acupuntura antes, durante e pós a gestação, bem como a incorporação e aplicabilidade dos resultados de estudos significativos na prática.

A pesquisa teve um percurso metodológico que se deu nas seguintes etapas: identificação do tema e seleção da hipótese ou questão de pesquisa; estabelecimento dos critérios de inclusão e exclusão das publicações (seleção da amostra); busca na literatura; análise e categorização dos estudos, apresentação e discussão dos resultados.

Para o levantamento dos artigos, os descritores foram selecionados a partir da terminologia em saúde consultada nos Descritores em Ciências da Saúde (DeCS). A busca foi realizada em dezembro de 2015 na Biblioteca Virtual em Saúde (BVS). Para a realização da pesquisa, foram utilizados os seguintes critérios de inclusão: cruzamento dos seguintes descritores: "Acupuntura" or "Medicina Tradicional Chinesa" and "Gestação" or "Gravidez" or "Obstetrícia", utilizando-se o método "busca avançada", "palavras no título"; foi realizada nos idiomas português e inglês; foram aplicados os filtros de busca somente por artigos e foram procurados estudos realizados no período de 2000 a 2015, caracterizados como artigos originais, revisões sistemáticas, relatos de experiências, ensaios teóricos e estudos de caso. Os critérios de exclusão adotados foram: duplicidade de estudos, não apresentar desenho metodológico claro ou apresentar outro tipo de terapia (moxabustão e auriculoterapia) concomitantemente acupuntura.

0 percurso metodológico encontra-se ilustrado na Figura 1.

\section{Resultados}

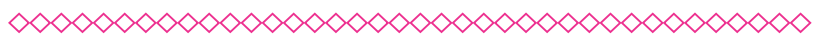

A busca primária gerou 57 artigos. Inicialmente, foram excluídos 14 por duplicidade, gerando 43 estudos para uma leitura detalhada. Após essa etapa, 20 artigos foram excluídos por não apresentarem abordagem metodológica clara ou apresentarem o uso concomitante de outro tipo de terapia. Dessa forma, 23 artigos foram selecionados e categorizados para a elaboração do presente estudo (Figura 1).

Dentre os artigos selecionados, seis correspondem a revisões bibliográficas ou sistemáticas, dois correspondem a estudos de caso e 15 artigos originais. Quanto às temáticas abordadas nos estudos, verificou-se que, da totalidade de artigos, independentemente do desenho metodológico: seis tratam de dor pélvica e lombar; sete, sobre a utilização da acupuntura e eventos adversos, incluindo aborto e indução ao parto; dois se referem à acupuntura sobre infertilidade e fertilização; cinco, sobre náuseas e vômito; dois, sobre ansiedade e depressão e um se refere à insônia. Todos tinham como objetivo comum avaliar a eficácia dessa técnica e verificar seus eventos adversos.

A leitura completa e minuciosa dos artigos possibilitou identificar cinco categorias temáticas: "utilização e eventos adversos da acupuntura relacionados à gestação", "acupuntura como abordagem na infertilidade feminina e processo de fertilização", "acupuntura como analgesia na dor pélvica e lombar durante a gestação", "acupuntura para náusea e vômito durante a gestação" e "acupuntura para depressão, ansiedade, irritabilidade, insônia durante e pós-gestação".

O Quadro 1 apresenta a síntese dos artigos, considerando autor(es), características metodológicas e principais contribuições.

\section{Discussão}

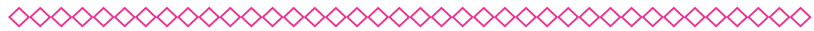

\section{Utilização e eventos adversos da acupuntura, relacionados à gestação}

A procura pela acupuntura durante a gestação ocorre por inúmeros motivos, por exemplo: preparar corpo e mente para o parto, reduzir a 
ansiedade e o estresse, facilitar ou manter a gravidez e o trabalho de parto, corrigir o mau posicionamento fetal, aliviar pirose, dores de forma geral, pré-eclâmpsia, controlar contrações falsas e problemas placentários, buscando equilibrar o sistema energético e evitar o tratamento convencional medicamentoso ${ }^{6}$. Entretanto, o número de estudos ainda é reduzido, assim como as evidências científicas que amparam o uso da acupuntura durante a gravidez ${ }^{7}$.

0 uso da acupuntura nos casos de gestação prolongada de 38 a 45 semanas apresentou tendência positiva. Todavia, a taxa de indução ao trabalho de parto demonstrou-se escassa para afirmar eficácia ${ }^{8}$. Além disso, não foram observados resultados positivos na aplicabilidade da acupuntura para indução ao trabalho de parto e retenção placentária; porém, constatou-se falta de eventos adversos e complicações pós-parto após seu uso em comparação e/ou associação a outros métodos da medicina alternativa e complementar ${ }^{9}$. Embora nesse estudo os autores não tenham investigado especificamente quais foram os métodos da medicina complementar associados à acupuntura, eles destacam que a utilização de homeopatias e aromaterapias, representam outros métodos da medicina alternativa e complementar observados em estudo anterior ${ }^{9}$.

Os eventos adversos graves encontrados na literatura não apresentam relação direta com a acupuntura, tais como: cordão umbilical preso ao pescoço ou ombro do feto. Enquanto outros eventos relacionados à prática são classificados como leves e moderados, por exemplo: sangramento local, hematoma, dor de cabeça, sonolência, piora do sintoma inicial, tontura, desconforto no ponto de inserção da agulha, equimose, contração uterina com ou sem dor abdominal, calor, sudorese, náusea, sensação de desmaio, distúrbio do sono, fadiga, irritabilidade, agitação, parestesia e edema ${ }^{10}$.

É complexo relacionar algumas complicações da gestação com reações da técnica de acupuntura. Descreve-se que, quando a complicação ocorre quatro ou mais semanas após a última sessão, descarta-se qualquer relação com a técnica. Certamente, a baixa quantidade e qualidade dos estudos relatando eventos adversos relacionados com a acupuntura durante a gestação limita uma conclusão mais sólida ${ }^{11}$.

Além dos questionamentos quanto à sua real aplicabilidade, a procura por efeitos adversos da acupuntura na gestação dá-se pelo entendimento alicerçado na literatura de base da MTC de que, alguns acupontos são proibidos neste período, devido à estimulação de energia na região uterina no sentido de descida e circulação de energia vital (Qi), que podem provocar a expulsão do feto antes do tempo. Nesse sentido, o uso dos pontos Baço Pâncreas 6 (BP6) e Intestino Grosso 3 (IG3) poderiam induzir contrações uterinas; Intestino grosso 4 (IG4) e BP6 podem provocar expulsão do feto durante o parto e nos casos de morte fetal; e Baço Pâncreas 4 (BP4) pode inibir o trabalho de parto prematuro ${ }^{12}$.

Alguns acupontos proibidos como o IG4, BP6 e pontos sacrais foram descritos como não apresentando efeitos negativos em sua aplicação, contextualizando a falta de argumentos aceitáveis para o entendimento deste processo. Entretanto, considera-se que embora não tenham sido apresentadas complicações relacionadas à acupuntura, a literatura clássica deve ser levada em consideração ${ }^{10}$.

As alterações fisiológicas no organismo da mulher após acupuntura, mais evidenciadas no uso da eletroacupuntura sobre a área lombar, são descritas como: aumento da oxigenação uterina através da diminuição na impedância da artéria uterina, a qual prejudica o desenvolvimento do embrião que naturalmente deve ocorrer em um ambiente de baixo oxigênio no primeiro trimestre de gestação. A acupuntura exerce efeito sobre 0 hipotálamo e hipófise, estimulando a secreção hormonal e interferindo nos níveis de progesterona, levantando questionamento quanto à influencia sobre contrações uterinas e expulsão do feto ${ }^{13}$.

A MTC propõe a acupuntura como estimulação energética para dentro do padrão energético normal do órgão afetado, buscando corrigir desequilíbrios no fluxo dos meridianos. Portanto, a estimulação cerebral e hormonal descrita não apresenta respaldo científico quanto a ter intensidade suficiente para complicações sobre o feto e a gestante ${ }^{13}$, como observado neste estudo. 


\section{Acupuntura na infertilidade feminina e processo de fertilização}

$\mathrm{Na}$ busca por intervenções para o processo de fertilização, a escolha do tipo de tratamento utilizado muitas vezes está relacionada com questões de eficácia, custo, facilidade de utilização e administração, bem como avaliação dos efeitos colaterais. As técnicas hoje envolvem tratamento médico para induzir ovulação, cirúrgico como laparoscopia, histeroscopia, reprodução assistida dentre outros que apresentam alto custo fazendo com que os casais busquem as terapias complementares ${ }^{14}$.

Atualmente a transferência embrionária por fertilização in vitro (FIV) vem sendo o tratamento mais bem-sucedido para a infertilidade e, para muitas pessoas, fornece a última possibilidade de gravidez; além disso, muitas vezes múltiplos ciclos são necessários para alcançar sucesso ${ }^{15}$.

Nessa perspectiva, diferentes alternativas podem ser usadas para potencializar as chances da FIV. Dentre essas alternativas, a literatura científica mostra que a acupuntura pode melhorar significativamente a taxa de nascidos vivos, sugerindo que a técnica aumenta as chances de gravidez clínica em $65 \%$ dos casos comparados com o grupo controle $^{16}$. Entretanto, não são todos os trabalhos científicos que apresentam eficácia relacionando acupuntura e fertilização. De sete artigos analisados em uma revisão sistemática, somente dois demonstraram melhora na taxa de gravidez. Um parece ter favorecido a taxa de nascidos vivos e quatro estudos não apresentaram relevância clíni$\mathrm{ca}$, reafirmando as controvérsias e efetividade da acupuntura ${ }^{15}$.

Nos processos de reprodução, a acupuntura interfere na liberação imediata de neurotransmissores, os quais, por sua vez, estimulam o hormônio liberador de gonadotrofinas influenciando assim 0 ciclo menstrual, a ovulação e, consequentemente a fertilização ${ }^{17}$, bem como estimulação da inervação simpática sobre o útero através da inibição e ativação do fluxo sanguíneo ${ }^{18}$.

A acupuntura, portanto, apresenta um efeito promissor para infertilidade e não demonstra ser inferior à terapia hormonal padrão; porém, os estudos são escassos e controversos, sendo necessárias mais pesquisas para tal afirmação ${ }^{19}$.

\section{Acupuntura na analgesia para dor pélvica e lombar durante a gestação}

Durante o período gestacional a dor lombar e pélvica é uma das queixas mais comuns apresentadas pelas mulheres, com uma prevalência de 48 a $76 \%$, mais comum entre o segundo e terceiro trimestre gestacional ${ }^{20}$. Isto ocorre devido a mudanças fisiológicas, anatômicas e posturais no organismo da mulher gestante como: hiperlordose lombar, relaxamento ligamentar no púbis e articulações sacro-ilíacas, gerando outras compensações posturais que, com o avançar da gestação, sobrecarregam as articulações e musculatura ${ }^{21}$.

A acupuntura como intervenção para estas afecções objetiva redução da dor mediante estímulo energético. 0 efeito analgésico da acupuntura e seus eventos adversos foram avaliados na aplicação para vários tipos de dor nociceptivas durante o último trimestre de gravidez, demonstrando que a intensidade da dor diminuiu em $60 \%$ das participantes da acupuntura e $14 \%$ no grupo controle, não sendo encontradas complicações sérias $^{22}$.

A dor lombar e pélvica gera incapacidade, deficiência global funcional, impede permanência na vida profissional levando à procura pelo uso de analgésicos. Dessa forma, a acupuntura - como meio terapêutico eficaz com respaldo científico consistente e positivo - torna-se essencial no tratamento da gestante 23-19. $^{2}$.

A diminuição da dor por meio da acupuntura é geralmente imediata podendo perdurar até seis semanas após o estímulo inicial. E, quando associada com eletroacupuntura, o alívio da dor pode perdurar até seis meses após o estímulo, tanto no repouso quanto nas atividades de vida diária. Por isso, recomenda-se a utilização da acupuntura por estimulação manual visando a interferir minimamente sobre a gestação ${ }^{24}$.

Na acupuntura a inibição da dor ocorre através de mecanismos complexos neuro-humorais, opiáceos endógenos e monoaminas, com evidência de ação depressiva sobre neurônios do corno dorsal espinhal ${ }^{25}$. Outros mecanismos potenciais são observados como modulação do sistema límbico hipotalâmico, ativação do córtex insular ipsilateral como efeito específico do agulhamento. Contudo, 
os mecanismos envolvidos nesse processo ainda não estão $\operatorname{claros}^{26}$.

Outros tratamentos para dor e funcionalidade como a fisioterapia são utilizados na fase gestacional, e quando comparados à acupuntura apresentaram respostas mais lentas. Em alguns casos a acupuntura teve efeito imediato, na primeira sessão, sendo significativamente menor o tempo para melhoria do quadro do que quando empregada fisioterapia incluindo calor, massagem e hidroterapia nas sessões ${ }^{27}$.

Entende-se, portanto, que a acupuntura demonstrou grande efeito na resolução da dor pélvica e lombar de gestantes, sendo mais eficaz quanto ao tempo para melhoria do quadro álgico do que outras terapias, e não demonstrando efeitos adversos significativos quando comparada a outras técnicas, como grupos placebo de acupuntura (sham), pois eventos como indução de parto, aumento da ocitocina, parto prematuro foram semelhantes nos grupos estudados quando ocorreram $^{27-28}$.

Quanto à técnica da acupuntura nas queixas de dor lombar e pélvica dos seis estudos avaliados, cinco apresentam protocolos como descrito na literatura de base com sessões de duração de 20 a 30 minutos. Um estudo seguiu protocolo diferente ao encontrado na literatura, porém alcançou bons resultados. Neste, as agulhas eram deixadas 30 a 60 segundos, eram removidas e os pacientes permaneciam em repouso por 10 minutos, para novamente serem reaplicadas as agulhas. 0 tratamento de acupuntura foi de no mínimo de seis e no máximo de 12 sessões, utilizando um número de quatro a seis pontos aplicados bilateralmente ou não.

\section{Acupuntura para náusea e vômito durante a gestação}

Episódios de náuseas e vômitos são sintomas comuns, experimentados por algumas mulheres no primeiro trimestre da gravidez, isso porque mudanças hormonais deste período costumam alterar a função normal do trato digestório, como alterações sobre os níveis séricos de estrógenos e gonadotrofina coriônica humana, bem como do sistema vestibular, do paladar e da sensibilidade olfativa ${ }^{29}$.
Náusea e vômito geralmente são sintomas passageiros; porém, a persistência destes sintomas ou início após a décima segunda semana de gestação é designado hiperemese gravídica que acarreta em consequências mais complexas a mãe e ao bebê $\hat{e}^{30}$.

A alta prevalência destas afeç̧ões de aproximadamente 50 a $80 \%$ das gestantes instiga a atuação de novas terapias, visto que o tratamento medicamentoso não é indicado durante a gestação. Por isso, tratamentos antieméticos, como acupressão ou acupuntura, estão sendo utilizados evidenciando o acuponto Pericárdio 6 (PC6) para estas afeç̧ões devido ao seu efeito específico sobre a função digestiva alta ${ }^{31}$.

Um fator importante e extremamente positivo na escolha do tratamento por acupuntura é a velocidade da redução dos sintomas, sem utilização de medicação. Ao comparar o uso da acupuntura tradicional com acupuntura somente em PC6, com acupuntura sham e grupo controle, observa-se que a acupuntura tradicional gera um efeito mais imediato seguida do uso da acupuntura somente em $P C 6^{32}$.

Nesse sentido, há evidências que apontam a eficácia da acupuntura na redução dos sintomas logo na primeira semana de atendimento, enquanto o grupo placebo de acupuntura sham teve resultado mais tardio, mas positivo na redução da náusea e vômito ${ }^{33}$.

Quanto à efetividade da acupuntura sham e acupuntura tradicional, verifica-se que a acupuntura tradicional apresenta efeito fisiológico de ativação cerebral específica, os quais não foram encontrados na acupuntura sham ${ }^{26}$. Entretanto, para tratamento de náuseas e vômitos houveram excelentes resultados, sendo idênticos entre as duas técnicas ${ }^{34}$.

A acupuntura nos seus variados âmbitos de utilização apresentou efeitos positivos e promissores no meio hospitalar, quando utilizada para redução de sintomas de dor, náusea e vômito decorrentes do uso da medicação. Neste caso, a acupuntura nos pontos IG4 e PC6 diminuíram significativamente a dor e náusea em curto período possibilitando redução da medicação até a alta hospitalar ${ }^{35}$. 
As pesquisas comprovaram a eficácia da técnica e poucos eventos adversos foram levantados, os pontos utilizados para acupuntura em náusea e vômito foram condizentes com a literatura de base tradicional da MTC.

Acupuntura para depressão, ansiedade, irritabilidade e insônia durante e pós-gestação

Afecções psicológicas e afetivas também são alterações presentes na fase gestacional. Nos primeiros meses de gestação, muitas mulheres experimentam sintomas como cansaço, letargia, instabilidade emocional, irritação, redução da libido. Já a ansiedade tende a afetar mais de 15\% dos $\operatorname{casos}^{36}$. A depressão possui uma prevalência de $3,5 \%$ a $11 \%$ de casos no período gestacional, ressaltando seu aparecimento nos dois últimos meses de gestação com maior intensidade do que no pós-parto, sendo o tratamento medicamentoso, causando grande impasse na resolução desta doença ${ }^{37}$.

A acupuntura surge novamente como método inovador e eficaz dentro das terapias complementares. Quando comparado, o uso da acupuntura com o tratamento convencional psicológico acompanhado de fitoterápico pode demonstrar que a acupuntura foi capaz de reduzir os sintomas e melhorar a qualidade de vida de mulheres grávidas $^{36}$. A acupuntura apresentou maior taxa de resposta no controle da depressão do que demais tratamentos, mostrando-se como tratamento seguro e eficaz durante a gestação para casos de depressão ${ }^{37}$. Além da eficácia no tratamento de depressão pré-natal, a acupuntura também reduz a insônia e melhora significativamente a qualidade do sono ${ }^{7}$.

Além da depressão, o quadro de insônia durante a gestação é outro fator de diminuição de qualidade de vida, visto que a dor nas costas, o alinhamento postural, a dificuldade respiratória na postura deitada, aumento da necessidade de urinar e movimento do feto tende a dificultar o sono tranquilo. A acupuntura representa um tratamento eficaz, pois anula a necessidade de medicação para estes casos, melhora a qualidade do sono e reduz o cansaço diurno ${ }^{38}$.
Seu mecanismo de ação contempla a estimulação da liberação de serotonina, podendo gerar melhoria sobre estados emocionais alterados, do estresse emocional principalmente por causa do seu efeito ansiolítico, observados já nas primeiras sessões. Alguns acupontos com estes objetivos podem ser citados: BP6, Estômago 36 (E36), Vaso da Concepção 17 (VC17), Pulmão 6 (P6) e Vaso Governador 20 (VG20), presentes nos artigos utilizados em nossa pesquisa e literatura de base da $\mathrm{MTC}^{39}$.

Os efeitos da acupuntura tornam-se ainda mais importantes quando comparados aos efeitos negativos que o uso de medicação traz durante a gravidez, pois medicações - por exemplo, inibidores seletivos da recaptação da serotonina - apresentam risco potencial para efeitos teratogênicos sobre o feto ${ }^{40}$.

\section{Conclusão}

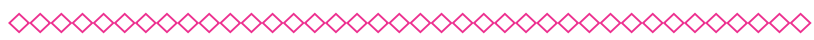

No período gestacional, a acupuntura demonstrou ser uma terapia eficaz e resolutiva na redução da dor, dos sintomas do sistema digestório alto, dos sintomas psicológicos, da infertilidade e trabalho de parto. Observou-se, também, que os mitos relacionados aos efeitos adversos e abortivos da acupuntura não foram identificados e, quando presentes, não houve comprovação da associação destes com a técnica.

Os dados disponíveis na literatura ainda são escassos e não proporcionam evidências consistentes e que possam possibilitar aos profissionais da área da saúde e obstetrícia maior segurança quanto aos efeitos da terapia por acupuntura, gerando intervenções mais assertivas capazes de engrandecer ou solucionar as afeç̧ões que se apresentam neste período. É de suma importância que sejam feitas novas pesquisas para sanar as dúvidas dos profissionais e possibilitar melhor acolhimento desta técnica pela população, visando a melhorar a qualidade de vida e saúde dos indivíduos. 


\section{Referências}

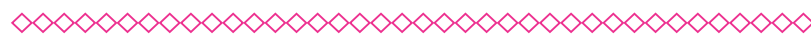

1. Spotorno PM, Silva IM, Lopes RS. Expectativas e sentimentos de mulheres em situação de reprodução medicamente assistida. Aletheia. 2008; 28:104-18.

2. Buchabqui JA, Abeche AM, Nickel C. Assistência pré-natal. In: Freitas F, Martins-Costa SH, Ramos JGL, Magalhães JA. Rotinas em obstetrícia. 5. ed. Porto Alegre: Artmed; 2006. p. 23-44.

3. Yamamura Y. Acupuntura tradicional: a arte de inserir. 2. ed. São Paulo: Roca; 2013.

4. Maciocia G. Os fundamentos da medicina chinesa: um texto abrangente para acupunturistas e fitoterapeutas. São Paulo: Roca; 2007.

5. Maciocia G. Diagnóstico na medicina chinesa: um guia geral. São Paulo: Roca; 2006.

6. Soliday E, Hapke P. Patient expectations of acupuncture in pregnancy. Glob Adv Health Med. 2014; 3(4):14-9.

7. Xu J, MacKenzie IZ. The current use of acupuncture during pregnancy and childbirth. Curr Opin Obstet Gynecol. 2012; 24(2):65-71.

8. Neri I, Monari F, Midwife CS, Facchinetti F. Acupuncture in post-date pregnancy: a pilot study. J Matern Fetal Neonatal Med. 2014; 27(9):874-8.

9. Münstedt K, Thienel J, Hrogovic I, Hackethal A, Kalder M, Misselwitz B. Use of acupuncture and other CAM methods in obstetrics: an analysis of 409.413 deliveries from Hesse, Germany. J Altern Complement Med. 2010; 17(5):421-6.

10. Park J, Sohn Y, White AR, Lee H. The safety of acupuncture during pregnancy: a systematic review. Acupunct Med. 2014; 32(3):257-66.

11. Clarkson CE, O'Mahony D, Jones DE. Adverse event reporting in studies of penetrating acupuncture during pregnancy: a systematic review. Acta Obstet Gynecol Scand. 2015; 94(5):453-64.
12. Silva VGS, Nakamura MU, Silva JBG. "Forbidden points" in pregnancy: do they exist? Acupunct Med. 2011; 29(2):135-6.

13. Cummings, M. Forbidden point's in pregnancy: no plausible mechanism for risk. Acupunct Med. 2011; 29(2):140-2.

14. Kamel, RM. Management of the infertile couple: an evidence-based protocol. Reprod Biol Endocrinol. 2010; (6):8-21.

15. Zheng $\mathrm{CH}$, Huang GY, Zhang MM, Wang $W$. Effects of acupuncture on pregnancy rates in women undergoing in vitro fertilization: a systematic review and meta-analysis. Fertil Steril. 2012; 97(3):599-611.

16. Manheimer $E$, Zhang G, Udoff L, Haramati $A$, Langenberg $P$, Berman BM, et al. Effects of acupuncture on rates of pregnancy and live birth among women undergoing in vitro fertilisation: systematic review and meta-analysis. Br Med J. 2008; 336(7643):545-9.

17. Stener-Victorin $E$, Waldenström $U$, Andersson SA, Wikland M. Reduction of blood flow impedance in the uterine arteries of infertile women with electro-acupuncture. Hum Reprod. 1996; 11(6):1314-7.

18. Cho ZH, Chung SC, Jones JP, Park JB, Park $\mathrm{HJ}$, Lee $\mathrm{HJ}$, et al. New findings of the correlation between acupoints and corresponding brain cortices using functional MRI. Proc Natl Acad Sci USA. 1998; 95(5):2670-3.

19. Ernst E, Lee MS, Choi TY. Acupuncture in obstetrics and gynecology: An overview of systematic reviews. The Americ Jof Chin Med. 2011; 39(3):423-431.

20. Silva JBG, Nakamura MU, Cordeiro JA, Kulay Jr L. Acupuncture for low back pain in pregnancy - a prospective, quasi-randomised, controlled study. Acupunct Med. 2004; 22(2):60-7.

21. Berg G, Hammar M, Möller-Nielsen J, Lindén ULF, Thorblad J. Low back pain during pregnancy. Obst Gynecol. 2008; 71(1):71-5.

22. Kvorning N, Holmberg C, Grennert L, Aberg A, Akeson J. Acupuncture relieves pelvic and low- 
back pain in late pregnancy. Acta Obstet Gynecol Scand. 2004; 83(3):246-50.

23. Carolyn CEE, Manheimer E, Pirotta MV, White AR. Acupuncture for pelvic and back pain in pregnancy: a systematic review. Am J Obstet Gynecol. 2008; 198(3):254-9.

24. Ternov NK, Grennert L, Aberg A, Algotsson L, Akeson J. Acupuncture for lower back and pelvic pain in late pregnancy: a retrospective report on 167 consecutive cases. Pain Med. 2001; 2(3):204-7.

25. Sandkuhler J. Learning and memory in pain pathways. Pain. 2000;88:113-8.

26. Pariente J, White P, Frackowiak RSJ, Lewith $G$. Expectancy and belief modulate the neuronal substrates of pain treated by acupuncture. NeuroImage. 2005; 25:1161-7.

27. Wedenberg K, Moen B, Norling A. A prospective randomized study comparing acupuncture with physiotherapy for low-back and pelvic pain in pregnancy. Acta Obstet Gynecol Scand. 2000; 79(5):331-5.

28. Elden $H$, Ostgaard HC, Fagevik-OIsen M, Ladfors $L$, Hagberg $\mathrm{H}$. Treatments of pelvic girdle pain in pregnant women: adverse effects of standard treatment, acupuncture and stabilising exercises on the pregnancy, mother, delivery and the fetus/neonate. BMC Complement Altern Med 2008; 8:34.

29. Coronado PJ, Fasero M, Alvarez-Sanchez Á, Rey E. Prevalence and persistence of nausea and vomiting along the pregnancy. Rev Esp Enferm Dig. 2014; 106(5):318-24.

30. Tachibana M, Duarte CAM, Santos LP, Lopes $\mathrm{MH}$. Hiperemese gravídica: estudo de caso dos aspectos psicológicos presentes na gestante. Psicol Hosp. 2006; 4(2):1-22.

31. Smith C, Crowther C, Beilby J. Acupuncture to treat nausea and vomiting in early pregnancy: a randomized controlled trial. Birth. 2002; 29(1):1-9.

32. Smith C, Crowther C, Beilby J. Pregnancy outcome following women's participation in a randomised controlled trial of acupuncture to treat nausea and vomiting in early pregnancy. Complement Ther Med. 2002; 10(2):78-83.
33. Smith C, Crowther $C$. The placebo response and effect of time in a trial of acupuncture to treat nausea and vomiting in early pregnancy. Complement Ther Med. 2002; 10(4):210-6.

34. Knight B, Mudge C, Openshaw S, White A, Hart A. Effect of acupuncture on nausea of pregnancy: a randomized, controlled trial. Obstet Gynecol. 2001; 97(2):184-8.

35. Oomman S, Liu D, Cummings M. Acupuncture for acute postoperative pain relief in a patient with pregnancy-induced thrombocytopenia-a case report. Acupunct Med. 2005; 23(2):83-5.

36. Silva JBG. Acupuncture for mild to moderate emotional complaints in pregnancy - a prospective, quasi-randomised, controlled study. Acupunct Med. 2007; 25(3):65-71.

37. Manber R, Schnyer RN, Allen JJ, Rush AJ, Blasey CM. Acupuncture: a promising treatment for depression during pregnancy. J Affect Disord. 2004; 83(1):89-95.

38. Silva JB, Nakamura MU, Cordeiro JA, Kulay $L J$. Acupuncture for insomnia in pregnancy $-a$ prospective, quasi-randomised, controlled study. Acupunct Med. 2005; 23(2):47-51.

39. Guimarães CM, Pinge MCM, Yamamura $Y$, Mello LEAM. Effects of acupuncture on behavioral, cardiovascular and hormonal responses in restraint-stressed Wistar rats. Braz J Med Biol Res. 1997; 30(12):1445-50.

40. Laine $K$, Heikkinen $T$, Ekblad U, Kero P. Effects of exposure to selective serotonin reuptake inhibitors during pregnancy on serotonergic symptoms in newborns and cord blood monoamine and prolactin concentrations. Arch Gen Psychiatry. 2003; 60(7):720-6. 


\section{Anexos}

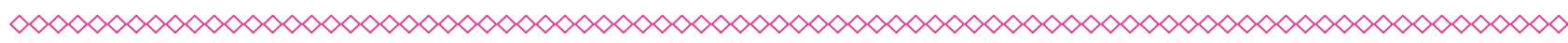

Figura 1 - Percurso metodológico

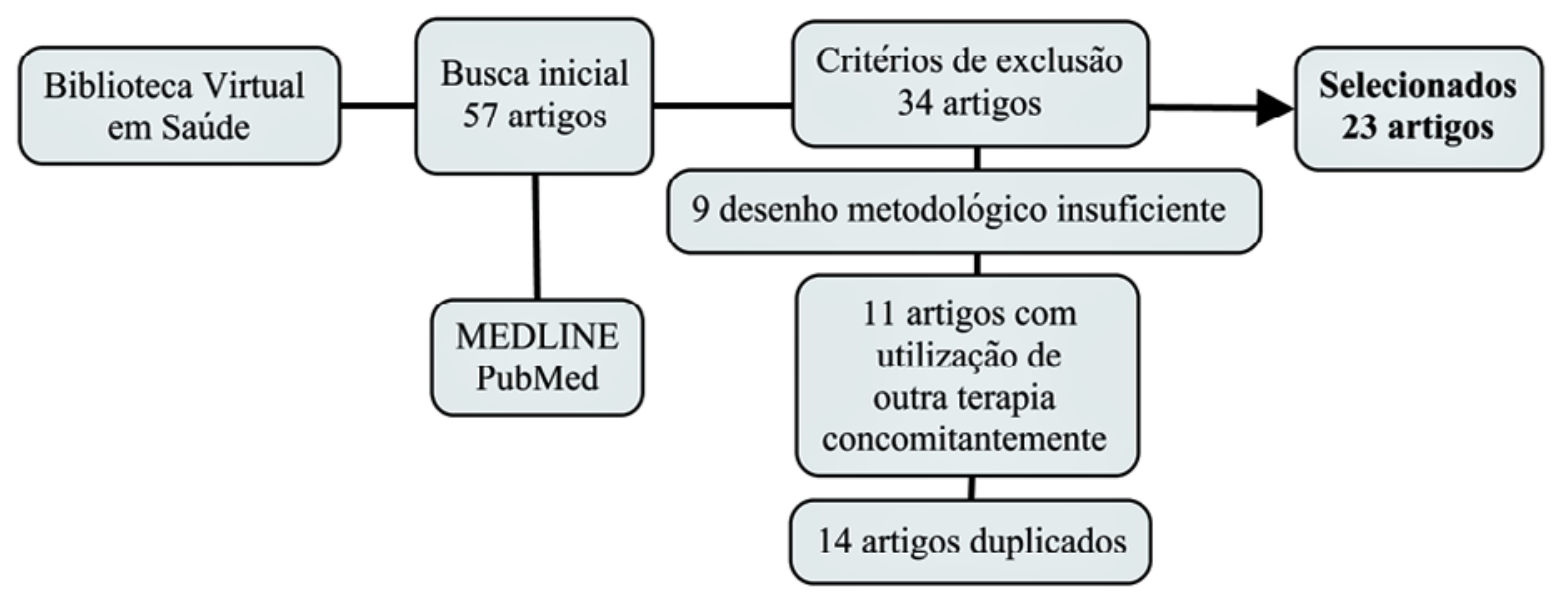

(clique para voltar ao texto)

Quadro 1 - Síntese dos artigos, segundo autor, características metodológicas e principais contribuições

\begin{tabular}{|c|c|c|c|c|c|}
\hline Autor & Tratamento & População & Metodologia & $\begin{array}{l}\text { Principais } \\
\text { Contribuições }\end{array}$ & $\begin{array}{l}\text { Pontos } \\
\text { Sugeridos }\end{array}$ \\
\hline \multicolumn{6}{|c|}{ Utilização e eventos adversos da acupuntura, relacionados à gestação } \\
\hline $\begin{array}{l}\text { Soliday, } \\
\text { Hapke }^{10}\end{array}$ & $\begin{array}{l}\text { Expectativas } \\
\text { frente ao uso } \\
\text { da acupuntura }\end{array}$ & $\begin{array}{c}265 \text { ex } \\
\text { pacientes }\end{array}$ & $\begin{array}{c}\text { Análise } \\
\text { retrospectiva }\end{array}$ & $\begin{array}{c}\text { Expectativas de } \\
\text { preparar corpo } \\
\text { e mente para o } \\
\text { parto, facilitar ou } \\
\text { manter a gravidez, } \\
\text { corrigir mau } \\
\text { posicionamento } \\
\text { do feto, induzir e } \\
\text { facilitar o trabalho } \\
\text { de parto, dores em } \\
\text { geral. }\end{array}$ & --- \\
\hline $\begin{array}{c}\text { Xu, } \\
\text { MacKenzie }{ }^{11}\end{array}$ & $\begin{array}{c}\text { Uso da } \\
\text { acupuntura } \\
\text { durante a } \\
\text { gravidez e o } \\
\text { parto }\end{array}$ & & $\begin{array}{c}\text { Estudo } \\
\text { randomizado }\end{array}$ & $\begin{array}{c}\text { Efeitos negativos } \\
\text { para hiperemese } \\
\text { gravídica, analgesia } \\
\text { de parto e indução } \\
\text { ao parto. Efeitos } \\
\text { positivos para } \\
\text { depressão pré-natal } \\
\text { e insônia. }\end{array}$ & $\begin{array}{l}\text { VB21, C3, } \\
\text { PC6, BP6, } \\
\text { R16, E37, } \\
\text { B67, IG4, } \\
\text { B32, B54, } \\
\text { B60 }\end{array}$ \\
\hline
\end{tabular}




\begin{tabular}{|c|c|c|c|c|c|}
\hline Autor & Tratamento & População & Metodologia & $\begin{array}{l}\text { Principais } \\
\text { Contribuições }\end{array}$ & $\begin{array}{l}\text { Pontos } \\
\text { Sugeridos }\end{array}$ \\
\hline Neri et al. ${ }^{12}$ & $\begin{array}{c}\text { Acupuntura } \\
\text { induzindo ao } \\
\text { parto }\end{array}$ & $\begin{array}{c}221 \\
\text { gestantes } \\
\text { entre } 40 \text { e } 46 \\
\text { semanas }\end{array}$ & Estudo piloto & $\begin{array}{l}\text { Não alterou a taxa } \\
\text { de indução ao } \\
\text { parto realizado } \\
\text { para a gravidez } \\
\text { prolongada, em } 45 \\
\text { semanas }\end{array}$ & $\begin{array}{c}\text { BP3, E36, } \\
\text { VB34, F3, } \\
\text { PC6, C7 }\end{array}$ \\
\hline $\begin{array}{l}\text { Münstedt et } \\
\text { al. }{ }^{13}\end{array}$ & $\begin{array}{c}\text { Uso da } \\
\text { acupuntura no } \\
\text { momento do } \\
\text { parto }\end{array}$ & $\begin{array}{c}409.413 \\
\text { partos }\end{array}$ & $\begin{array}{l}\text { Análise } \\
\text { retrospectiva }\end{array}$ & $\begin{array}{l}\text { Caracteriza } \\
\text { acupuntura como } \\
\text { método seguro, } \\
\text { em ascensão } \\
\text { junto a métodos } \\
\text { alternativos e } \\
\text { complementares. }\end{array}$ & --- \\
\hline Park et al. ${ }^{14}$ & $\begin{array}{c}\text { Eventos } \\
\text { adversos } \\
\text { associados ao } \\
\text { tratamento de } \\
\text { acupuntura }\end{array}$ & 105 artigos & $\begin{array}{c}\text { Revisão } \\
\text { sistemática }\end{array}$ & $\begin{array}{l}\text { Constatou que } \\
\text { eventos adversos } \\
\text { associado com a } \\
\text { acupuntura são } \\
\text { geralmente leves e } \\
\text { os graves são raros. }\end{array}$ & --- \\
\hline $\begin{array}{l}\text { Clarkson, } \\
\text { O'Mahony, } \\
\text { Jones }^{15}\end{array}$ & $\begin{array}{c}\text { Eventos } \\
\text { adversos da } \\
\text { acupuntura na } \\
\text { gravidez }\end{array}$ & 17 artigos & $\begin{array}{l}\text { Revisão } \\
\text { sistemática }\end{array}$ & $\begin{array}{l}\text { Baixa qualidade dos } \\
\text { artigos publicados. }\end{array}$ & --- \\
\hline \multicolumn{6}{|c|}{ Acupuntura na infertilidade feminina e processo de fertilização } \\
\hline $\begin{array}{l}\text { Ernst, Lee, } \\
\text { Choi }^{19}\end{array}$ & $\begin{array}{c}\text { Acupuntura } \\
\text { e afecções } \\
\text { ginecológicas e } \\
\text { obstétricas }\end{array}$ & 24 artigos & $\begin{array}{l}\text { Revisão } \\
\text { sistemática }\end{array}$ & $\begin{array}{c}\text { Nove estudos } \\
\text { apresentaram } \\
\text { efeitos positivos } \\
\text { da acupuntura, } \\
\text { mas salienta-se a } \\
\text { baixa qualidade dos } \\
\text { estudos. }\end{array}$ & --- \\
\hline $\begin{array}{l}\text { Zheng et } \\
\text { al. }^{20}\end{array}$ & $\begin{array}{l}\text { Infertilidade e } \\
\text { fertilização in } \\
\text { vitro }\end{array}$ & $\begin{array}{c}24 \text { artigos, } \\
5.807 \\
\text { participantes }\end{array}$ & $\begin{array}{c}\text { Revisão } \\
\text { sistemática e } \\
\text { meta análise }\end{array}$ & $\begin{array}{l}\text { A acupuntura } \\
\text { pode melhorar a } \\
\text { taxa de gravidez } \\
\text { clínica e a taxa } \\
\text { de nascidos vivos } \\
\text { entre mulheres } \\
\text { submetidas à } \\
\text { fertilização in } \\
\text { vitro, porém } \\
\text { reafirma as grandes } \\
\text { controvérsias. }\end{array}$ & --- \\
\hline
\end{tabular}




\begin{tabular}{|c|c|c|c|c|c|}
\hline Autor & Tratamento & População & Metodologia & $\begin{array}{c}\text { Principais } \\
\text { Contribuições }\end{array}$ & $\begin{array}{l}\text { Pontos } \\
\text { Sugeridos }\end{array}$ \\
\hline $\begin{array}{c}\text { Manheimer } \\
\text { et al. }{ }^{21}\end{array}$ & $\begin{array}{c}\text { Infertilidade e } \\
\text { fertilização in } \\
\text { vitro }\end{array}$ & $\begin{array}{c}7 \text { artigos, } \\
1366 \\
\text { participantes }\end{array}$ & $\begin{array}{c}\text { Revisão } \\
\text { sistemática e } \\
\text { meta análise }\end{array}$ & $\begin{array}{c}\text { Sugere que a } \\
\text { acupuntura } \\
\text { aumenta as chances } \\
\text { de gravidez em } \\
65 \% \text {, se comparadas } \\
\text { ao grupo controle. }\end{array}$ & --- \\
\hline \multicolumn{6}{|c|}{ Acupuntura na analgesia para dor pélvica e lombar durante a gestação } \\
\hline $\begin{array}{l}\text { Ernst, Lee, } \\
\text { Choi }^{19}\end{array}$ & $\begin{array}{c}\text { Acupuntura } \\
\text { e afecções } \\
\text { ginecológicas e } \\
\text { obstétricas }\end{array}$ & 24 artigos & $\begin{array}{c}\text { Revisão } \\
\text { sistemática }\end{array}$ & $\begin{array}{c}\text { Nove estudos } \\
\text { apresentaram } \\
\text { efeitos positivos } \\
\text { da acupuntura, } \\
\text { porém salienta a } \\
\text { baixa qualidade } \\
\text { metodológica dos } \\
\text { estudos. }\end{array}$ & --- \\
\hline Silva et al. ${ }^{25}$ & $\begin{array}{l}\text { Dor lombar e } \\
\text { pélvica }\end{array}$ & 61 gestantes & $\begin{array}{c}\text { Estudo } \\
\text { prospectivo } \\
\text { controlado } \\
\text { quasi- } \\
\text { randomizado }\end{array}$ & $\begin{array}{c}\text { A acupuntura } \\
\text { parece ser eficaz } \\
\text { para reduzir } \\
\text { os sintomas } \\
\text { e melhorar a } \\
\text { qualidade de } \\
\text { vida de mulheres } \\
\text { grávidas com } \\
\text { segurança. }\end{array}$ & $\begin{array}{l}\text { R13, ID3, } \\
\text { B62, B40, } \\
\text { TA5, VB30, } \\
\text { VB41 e } \\
\text { pontos } \\
\text { Huatojiaji }\end{array}$ \\
\hline $\begin{array}{c}\text { Kvorning et } \\
\text { al. }{ }^{27}\end{array}$ & $\begin{array}{c}\text { Dor lombar e } \\
\text { pélvica }\end{array}$ & 72 gestantes & $\begin{array}{c}\text { Estudo } \\
\text { prospectivo } \\
\text { randomizado }\end{array}$ & $\begin{array}{c}\text { Conclui que a } \\
\text { intensidade da dor } \\
\text { diminuiu em } 60 \% \\
\text { das participantes da } \\
\text { acupuntura e } 14 \% \\
\text { no grupo controle. }\end{array}$ & $\begin{array}{l}\text { F3, VG20, } \\
\text { B60, ID3, } \\
\text { B22, B26 }\end{array}$ \\
\hline $\begin{array}{l}\text { Carolyn et } \\
\text { al. }{ }^{28}\end{array}$ & $\begin{array}{c}\text { Dor lombar e } \\
\text { pélvica }\end{array}$ & 3 artigos & $\begin{array}{c}\text { Revisão } \\
\text { sistemática }\end{array}$ & $\begin{array}{l}\text { Efeitos positivos } \\
\text { sobre alívio da dor } \\
\text { pela acupuntura. }\end{array}$ & --- \\
\hline $\begin{array}{c}\text { Ternov et } \\
\text { al. }^{29}\end{array}$ & $\begin{array}{l}\text { Dor lombar e } \\
\text { pélvica }\end{array}$ & $\begin{array}{c}167 \\
\text { gestantes }\end{array}$ & $\begin{array}{c}\text { Estudo } \\
\text { retrospectivo }\end{array}$ & $\begin{array}{l}\text { Produziu analgesia } \\
\text { na dor lombar; } \\
\text { seis semanas } \\
\text { após o início da } \\
\text { estimulação, } \\
\text { observou que } \\
\text { associar eletro } \\
\text { acupuntura de } \\
\text { baixa frequência } \\
\text { proporcionou alívio } \\
\text { da dor persistente. }\end{array}$ & $\begin{array}{c}\text { F3, IG4, BP9, } \\
\text { VG20, B57, } \\
\text { B60, E36, } \\
\text { ID3, VB34 }\end{array}$ \\
\hline
\end{tabular}




\begin{tabular}{|c|c|c|c|c|c|}
\hline Autor & Tratamento & População & Metodologia & $\begin{array}{l}\text { Principais } \\
\text { Contribuições }\end{array}$ & $\begin{array}{l}\text { Pontos } \\
\text { Sugeridos }\end{array}$ \\
\hline $\begin{array}{c}\text { Wedenberg, } \\
\text { Moen, } \\
\text { Norling }^{32}\end{array}$ & $\begin{array}{c}\text { Dor lombar e } \\
\text { pélvica }\end{array}$ & 60 gestantes & $\begin{array}{c}\text { Estudo } \\
\text { prospectivo } \\
\text { randomizado }\end{array}$ & $\begin{array}{c}\text { O efeito da } \\
\text { acupuntura em } \\
\text { alguns casos foi } \\
\text { imediato, sendo } \\
\text { significativamente } \\
\text { em menor tempo } \\
\text { do que o grupo } \\
\text { que realizou } \\
\text { fisioterapia. }\end{array}$ & $\begin{array}{c}\text { B26, B27, } \\
\text { B28, B29, } \\
\text { B30, B60, } \\
\text { CV2 e pontos } \\
\text { locais }\end{array}$ \\
\hline Elden et al. ${ }^{33}$ & $\begin{array}{c}\text { Dor lombar e } \\
\text { pélvica }\end{array}$ & $\begin{array}{c}386 \\
\text { gestantes }\end{array}$ & Ensaio clínico & $\begin{array}{c}\text { Redução na } \\
\text { intensidade da } \\
\text { dor, não relatando } \\
\text { eventos adversos. }\end{array}$ & $\begin{array}{l}\text { VG20, IG4, } \\
\text { R11, E36, } \\
\text { BP2, VB30, } \\
\text { B26, B32, } \\
\text { B33, B54, } \\
\text { B60, EX21 }\end{array}$ \\
\hline \multicolumn{6}{|c|}{ Acupuntura para náusea e vômito durante a gestação } \\
\hline $\begin{array}{l}\text { Smith, } \\
\text { Crowther, } \\
\text { Beilby }^{36}\end{array}$ & $\begin{array}{l}\text { Náusea e } \\
\text { vômito }\end{array}$ & $\begin{array}{c}593 \\
\text { gestantes }\end{array}$ & $\begin{array}{c}\text { Estudo } \\
\text { randomizado } \\
\text { controlado }\end{array}$ & $\begin{array}{c}\text { A acupuntura } \\
\text { demonstrou eficácia } \\
\text { na redução de } \\
\text { náusea e vômito no } \\
\text { início da gravidez. }\end{array}$ & $\begin{array}{c}\text { R21, R22, } \\
\text { E19, E20, } \\
\text { E21, E34, } \\
\text { E36, E40, } \\
\text { E44, VC10, } \\
\text { VC11, VC12, } \\
\text { VC13, VC14, } \\
\text { P6, PC3, C5, } \\
\text { VB34, BP9, } \\
\text { B20, B15 }\end{array}$ \\
\hline $\begin{array}{l}\text { Smith, } \\
\text { Crowther, } \\
\text { Beilby }^{37}\end{array}$ & $\begin{array}{l}\text { Náusea e } \\
\text { vômito }\end{array}$ & $\begin{array}{c}583 \\
\text { gestantes }\end{array}$ & $\begin{array}{c}\text { Estudo } \\
\text { randomizado } \\
\text { controlado }\end{array}$ & $\begin{array}{c}\text { A acupuntura } \\
\text { tradicional } \\
\text { apresentou efeitos } \\
\text { mais imediatos } \\
\text { do que a terapia } \\
\text { utilizando } \\
\text { PC6, ambos os } \\
\text { resultados eficazes. }\end{array}$ & $\begin{array}{c}\text { E19, E20, } \\
\text { E21, R20, } \\
\text { R21, VC10, } \\
\text { VC11, VC12, } \\
\text { VC13, VC14 } \\
\text { e um grupo } \\
\text { somente PC6 }\end{array}$ \\
\hline $\begin{array}{c}\text { Smith, } \\
\text { Crowther }^{38}\end{array}$ & $\begin{array}{l}\text { Náusea e } \\
\text { vômito }\end{array}$ & $\begin{array}{c}593 \\
\text { gestantes }\end{array}$ & $\begin{array}{c}\text { Estudo } \\
\text { randomizado } \\
\text { controlado }\end{array}$ & $\begin{array}{l}\text { O maior efeito } \\
\text { terapêutico para } \\
\text { reduzir náuseas e } \\
\text { vômito seco veio } \\
\text { de acupuntura } \\
\text { tradicional na } \\
\text { primeira semana. }\end{array}$ & $\begin{array}{c}\text { E19, E20, } \\
\text { E21, R20, } \\
\text { R21, VC10, } \\
\text { VC11, VC12, } \\
\text { VC13, VC14 } \\
\text { e um grupo } \\
\text { somente PC6 }\end{array}$ \\
\hline
\end{tabular}




\begin{tabular}{|c|c|c|c|c|c|}
\hline Autor & Tratamento & População & Metodologia & $\begin{array}{l}\text { Principais } \\
\text { Contribuições }\end{array}$ & $\begin{array}{l}\text { Pontos } \\
\text { Sugeridos }\end{array}$ \\
\hline $\begin{array}{l}\text { Knight } \\
\text { et al. }{ }^{39}\end{array}$ & $\begin{array}{l}\text { Náusea e } \\
\text { vômito }\end{array}$ & 55 gestantes & $\begin{array}{c}\text { Estudo } \\
\text { randomizado } \\
\text { controlado }\end{array}$ & $\begin{array}{l}\text { A partir de três } \\
\text { sessões de } \\
\text { acupuntura ou } \\
\text { acupuntura sham, } \\
\text { houve diminuições } \\
\text { notáveis em } \\
\text { náusea da gravidez } \\
\text { precoce, porém sem } \\
\text { diferença entre os } \\
\text { grupos. }\end{array}$ & $\begin{array}{l}\text { E34, E36, } \\
\text { E44, VC12, } \\
\text { BP4, PC6 }\end{array}$ \\
\hline $\begin{array}{l}\text { Oomman, } \\
\text { Liu, } \\
\text { Cummings }\end{array}$ & $\begin{array}{l}\text { Dor e náusea } \\
\text { decorrente de } \\
\text { medicação }\end{array}$ & 1 gestante & $\begin{array}{l}\text { Estudo de } \\
\text { caso }\end{array}$ & $\begin{array}{l}\text { O alívio da dor, } \\
\text { náusea e angústía } \\
\text { parece reduzir logo } \\
\text { após a inserção } \\
\text { das agulhas da } \\
\text { acupuntura. }\end{array}$ & IG4, PC6 \\
\hline \multicolumn{6}{|c|}{ Acupuntura para depressão, ansiedade, irritabilidade e insônia durante e pós-gestação } \\
\hline Silva ${ }^{41}$ & $\begin{array}{c}\text { Queixas } \\
\text { emocionais leve } \\
\text { e moderada }\end{array}$ & 51 gestantes & $\begin{array}{c}\text { Estudo } \\
\text { prospectivo } \\
\text { controlado } \\
\text { quasi- } \\
\text { randomizado }\end{array}$ & $\begin{array}{l}\text { A acupuntura foi } \\
\text { capaz de reduzir } \\
\text { os sintomas } \\
\text { e melhorar a } \\
\text { qualidade de } \\
\text { vida de mulheres } \\
\text { grávidas. }\end{array}$ & $\begin{array}{c}\text { C7, PC6, } \\
\text { P9, E36, F3, } \\
\text { VG20, VC17 }\end{array}$ \\
\hline $\begin{array}{l}\text { Manber } \\
\text { et al. }{ }^{42}\end{array}$ & $\begin{array}{l}\text { Depressão e } \\
\text { ansiedade }\end{array}$ & 61 gestantes & $\begin{array}{l}\text { Estudo piloto, } \\
\text { randomizado } \\
\text { controlado }\end{array}$ & $\begin{array}{l}\text { A acupuntura é uma } \\
\text { promessa como } \\
\text { um tratamento } \\
\text { seguro e eficaz para } \\
\text { depressão durante } \\
\text { a gravidez. }\end{array}$ & $\begin{array}{c}\text { IG4, BP1, } \\
\text { BP6, VB21, } \\
\text { B60, B67, } \\
\text { VC3, VC4, } \\
\text { VC5, VC6, } \\
\text { E36, E45, } \\
\text { B23, B32, R4, } \\
\text { VB44, VC12 }\end{array}$ \\
\hline Silva et al. ${ }^{43}$ & $\begin{array}{l}\text { Insônia durante } \\
\text { a gestação }\end{array}$ & 30 gestantes & $\begin{array}{c}\text { Estudo } \\
\text { prospectivo } \\
\text { controlado } \\
\text { quasi- } \\
\text { randomizado }\end{array}$ & $\begin{array}{l}\text { A acupuntura foi } \\
\text { eficaz, anulando } \\
\text { a necessidade de } \\
\text { medicação para } \\
\text { estes casos. }\end{array}$ & $\begin{array}{c}\text { C7, PC6, } \\
\text { VB21, extra } \\
\text { Anmiane } \\
\text { Yintang, } \\
\text { VG20, VC17 }\end{array}$ \\
\hline
\end{tabular}

